\title{
Stress-Induced Premature Senescence
}

\section{Essence of Life, Evolution, Stress, and Aging}

\author{
OLIVIER TOUSSAINT, ${ }^{b}$ PATRICK DUMONT, JEAN-FRANÇOIS DIERICK, \\ THIERRY PASCAL, CHRISTOPHE FRIPPIAT, FLORENCE CHAINIAUX, \\ FRANCIS SLUSE, ${ }^{a}$ FRANÇOIS ELIAERS, AND JOSÉ REMACLE \\ Department of Biology, Unit of Cellular Biochemistry and Biology, \\ University of Namur (FUNDP), B- 5000 Namur, Belgium \\ ${ }^{a}$ Laboratory of Bioenergetics, University of Liège, B-4000 Liège (Sart-Tilman), Belgium
}

\section{INTRODUCTION. WHAT IS STRESS?}

The stress syndrome was discovered accidentally by Hans Selye while searching for new hormones in the placenta. ${ }^{1}$ After injecting rats with crude preparations, Selye found adrenal enlargements and involution of thymus and lymph nodes, which he thought were specific for a particular hormone. It occurred to Selye that these symptoms might represent a nonspecific response to noxious agents. Indeed, this was found to be the case when he injected rats with diverse agents. Selye defined the stress response as the "general adaptation syndrome."2,3 According to this theory, the initial reaction to stress is shock, it is followed by a countershock phase, and gradually resistance develops to the stressor. This resistance may turn into exhaustion, however, if the stressor persists, and death may ensue. Both specific and nonspecific resistance develops during stress. ${ }^{4}$ In his last scientific book, Selye defined biologic stress as "the non-specific response of the body to any demand made upon it." 5 Beside the transfer of the word "stress" from physics to biology, Selye also coined the words corticosteroids, glucocorticoids, and mineralocorticoids. ${ }^{6}$ Nowadays, the concept of stress has invaded most fields of the biologic, medical, and social sciences. Cellular and molecular biology has become interested in the study of the stress response of human, animal, and plant cells, the consensus being that "any environmental factor potentially unfavourable to living organism" is stress. ${ }^{7}$ It is also generally agreed that "if the limits of tolerance are exceeded and the adaptive capacity is over-worked, the result may be permanent damage or even death." 8 Three phases of the stress response have been defined based on experimental observations: (1) the response phase of alarm reaction with deviation of functional norm, decline of vitality, and excess of catabolic processes over anabolism, (2) the restitution phase or stage of resistance with adaptation processes and repair processes, and (3) either the end phase, that stage of exhaustion or long-term response when stress intensity is too high, leading to overcharge of the adaptation capacity, damage, chronic dis-

\footnotetext{
${ }^{b}$ Corresponding author: Olivier Toussaint, Department of Biology, Unit of Cellular Biochemistry \& Biology, University of Namur (FUNDP), Rue de Bruxelles, 61, B-5000 Namur, Belgium. Phone: +32 817241 32; fax: +32 81724135 .

olivier.toussaint@fundp.ac.be
} 
ease, senescence, or even death, or the regeneration phase, full restoration of physiologic function, when the stressor is removed. ${ }^{9}$

\section{STRESS AND LIFE SPAN}

It has been proposed that a subset of genes, termed "public," may be of particular importance in how most individuals of a species age, whereas "private" mechanisms of aging would control the rate of aging of particular individuals. ${ }^{10}$ It has also been proposed that genes involved in the stress response, such as genes coding for DNA repair enzymes and antioxidant enzymes, could be among these genes regulating aging.

A theory analogically derived from the darwinian theory of evolution proposed that organisms (or cells from organisms) that have the longest life span should secure their longevity through investment in a more durable soma and including enhanced cellular resistance to stress, arguing that long life span means evolution-driven better adaptation to stress. To investigate whether cells from long-lived species have better mechanisms to cope with oxidative and nonoxidative stress, the survival of primary skin fibroblasts and lymphocytes from eight mammalian species with a range of life spans was estimated following stresses induced by oxidative and alkaline stress. Cellular resistance to the stresses tested was positively correlated with mammalian longevity. ${ }^{11}$

The evolutionary theory of aging suggests that senescence occurs because the force of natural selection declines with age and because longevity is only acquired at some metabolic cost. Organisms may trade late survival for enhanced reproductive investments in earlier life. ${ }^{12,13}$ It has been proposed in the "network theory of aging" that "an important corollary of this hypothesis is that multiple mechanisms of aging operate in parallel," 14 thereby connecting this theory to other theories of aging. For instance, the theory of catastrophic error proposed by Orgel ${ }^{15}$ argued that aging is caused by the accumulation of many kinds of defects. The "global concept of failure of maintenance," proposed by Holliday, ${ }^{16}$ and the concept of "critical threshold"17,18 were the basis for the network theory of aging. Indeed, these theories proposed that aging could be a consequence of the accumulation of defects, thereby including all "wear and tear" theories such as the theory of free radicals and the mitochondrial theory of aging. In those theories, it was also postulated that this accumulation of defects may be due not only to an increase in damage generation but also to a decrease in the efficiency of elimination and repair systems such as the antioxidant system, the system responsible for the degradation of altered proteins, DNA repair systems, etc. Theoretical developments based on a model of free radical production and elimination also confirmed the possibility of a threshold beyond which the cellular system is unstable. ${ }^{18}$

The fundamental question is why do cells, during aging, undergo internal modifications either spontaneously due to molecular decay or due to interactions with "any environmental factor potentially unfavourable to living organisms," that is to say, stress (oxidative, mechanical, heat, etc), reaching finally a threshold of error leading to irreversible degeneration and death. The answer to this question is probably linked to why and how cells exist and evolve. Therefore, to propose an answer 
to this question, it was necessary to use a theoretical background, considering the very essence of living matter, in order to avoid analogical extrapolations about the theory of aging. This question was approached by using the thermodynamics of open systems, which allows a global systemic description of the cell's behavior and, in this way, transcends the genetic or stochastic considerations of the aging process as well as evolutionary questions.

\section{THE ESSENCE OF LIFE AND AGING}

The first law of thermodynamics states that energy cannot be created or destroyed and that the total energy within a closed system remains unchanged. The second law of thermodynamics requires that any process underway in a system irreversibly degrades the quality of the energy in that system in a direction that results in an entropy increase.

Let us focus our attention towards the class of thermodynamic systems that are open, moved away from equilibrium by the fluxes of material and energy across their boundary, and maintain their form or structure by continuous exchanges. This is done at a cost of increasing the entropy of the larger "global" system which contains such systems, because, following the second law, that overall entropy in the global sense must increase. Dissipative systems such as living systems, from cells to ecosystems, and nonliving organized systems, such as convection cells, tornadoes and lasers, are dependent on outside energy fluxes to maintain their organization in a locally reduced entropy state. The entropy relationships in dissipative systems were put forward by Denbeigh ${ }^{19}$ and Prigogine ${ }^{20}$ in the following relationship: $d S=d S_{\mathrm{i}}+$ $d S_{\mathrm{e}}$, where $d S$ is the total entropy change in a system and $d S_{\mathrm{i}}$ is the internal entropy in the system. According to the second law, $d S_{\mathrm{i}}$ is always greater than or equal to zero. $d S_{\mathrm{e}}$ is the entropy exchange with the environment, which may be positive, negative, or zero. For the system to maintain itself in a nonequilibrium steady state, $d S_{\mathrm{e}}$ must be negative and larger than the entropy produced by internal processes, $d S_{\mathrm{i}}$, that is, metabolism in the case of living cells.

Implicit in the observation that a system will tend towards its unique equilibrium state is that a system will also resist being removed from the equilibrium state. The degree to which a system has been moved from equilibrium can be measured by the gradients imposed on the system. As systems are moved away from equilibrium, they will utilize all avenues available to counter the applied gradients. Attractors can emerge so that the system organizes in a way that reduces or degrades the gradient. As the applied gradients increase, so does the system's ability to oppose further movement from equilibrium. If dynamic and/or kinetic conditions permit, self-organization processes are to be expected. The building of organizational structure and associated processes degrades the imposed gradient more effectively than if the dynamic and kinetic pathways for those structures were unavailable. ${ }^{21,22}$

This corollary of the second law holds for all living systems from cells to ecosystems and describes their expected behavior. Prigogine and others have shown that dissipative structures self-organize through fluctuations and instabilities that lead to irreversible bifurcations and new stable system states. Dissipative structures are stable over a finite range of conditions and are sensitive to fluxes and flows from out- 
side the system. Glansdorff and Prigogine ${ }^{23}$ have shown that these thermodynamic relationships can be represented by coupled nonlinear relationships, that is, autocatalytic positive feedback cycles, many of which lead to stable macroscopic structures that exist away from the equilibrium state. Convection cells, hurricanes, autocatalytic chemical reactions, and living systems are all examples of nonequilibrium dissipative structures that exhibit coherent behavior. Living cells are dissipative structures exhibiting processes of informed, self-replicating, autocatalytic cycles. This paradigm provides for a thermodynamically consistent explanation of why there is life, including the origin of life, biological growth, development of ecosystems, patterns of biological evolution observed in the fossil record, stress response, and aging. $21,22,24,25$

Maintenance of life, ontogenic (growth, development, and differentiation) and phylogenic evolution of living processes, from cells to ecosystems, is the response to the thermodynamic imperative of dissipating gradients. Biological growth occurs when the system adds more of the same types of pathways for degrading imposed gradients. Biological development occurs when new types of pathways emerge in the system for degrading imposed gradients. In biological terms, growth means an increase in the amount of substrates entering preexisting metabolic pathways, whereas a new step of development means the appearance of supplementary pathways. This can explain, for instance, the phylogenic appearance of more and more complex multicellular organisms with more and more complex metabolic pathways using and degrading the energy present in their environment, thus optimizing the production and use of free energy in function of the available substrate. ${ }^{21,22,24,25}$

At the present stage of evolution, mechanisms exist, such as predation, parasitism, infectious diseases, aging, and age-linked risks of getting sick, being caught by a predator, or starving, that lead to a decrease in the number of individuals of a species. We have seen that a possible criterion of growth, differentiation, development, and evolution, is the capacity of cells and organisms to degrade energy gradients from the environment, which is the capacity to produce more entropy per unit of time and moles of molecules entering the cellular system (i.e., input fluxes) and per unit of volume or weight of the system considered. A direct corollary is that this capacity decreases with aging, which is experimentally fulfilled. From this corollary, it can be predicted that evolution, which arrow of time depends on thermodynamics as we have seen, has selected mechanisms that are triggered when the capacity of the organisms to degrade energy starts to decrease and leads to the death of the organisms. 25,26

\section{STRESS AND GENOMES}

A modification of the balance between the capacities of repairing the damage caused by stresses and the accumulation of damage due to stress might be a factor responsible for this decrease in the capacity of gradient degradation.

In the thermodynamic model of aging, stresses are sorted into three different types depending on the ratio between the damage they generate and the capacity of stress response of the biological system considered. The thermodynamic development has been given in reference 26 . First, let us consider the constant, unavoidable, 
ubiquitous mild stresses such as steady state concentrations of reactive oxygen species in normal conditions. Thermodynamically these mild stresses do not immediately alter the stability of the system. However, the possibility remains for internal modifications to accumulate without immediately altering the cellular stability as long as they can be counterbalanced by other cellular mechanisms. This is the only possibility that allows the prediction that cells shift to a new steady state when the level of damage has reached a critical threshold where compensation mechanisms become momentarily overwhelmed. This new steady state is characterized by a higher level of damage and lower global metabolic activity. ${ }^{26}$ This is normal aging.

The second type of stress is chronic stress or (repeated) stress of sublethal intensity resulting from abnormal situations such as inflammation, anoxia-reoxygenation, and exposure to radioactivity, UV irradiation, and toxic chemicals. During such stress, the level of damage may rise so much that the capacity for a specific stress response is momentarily overwhelmed and damage spreads to various cell components, requiring other defense systems. If these systems can eliminate/repair all the damage, then the cell remains stable. When defense systems cannot eliminate/repair quickly all the damage, this may lead to a transient increase in the level of damage and a transient decrease in the capacity to produce (ATP, redox potential, etc.) and use (biochemical reactions) free energy. Such conditions represent the thermodynamic conditions for destabilization of the steady state of far from equilibrium systems and straightforwardly of living cells. Thermodynamically speaking, if the cell can reorganize at new steady state, there will be two major irreversible differences between this new steady state and the previous one, that is, a higher level of internal damage and lower global metabolic activity. These conditions lead to stress-induced premature senescence (SIPS). In this perspective, normal and accelerated aging would be ways for decreasing the metabolic activity of those cells that have a given level of damage, possibly to avoid cell transformation and premature decrease in the capacity of the organism to degrade energy gradients. It is also possible that depending on the cell type, the increase in the level of damage will trigger programmed mechanisms of self-destruction rather than accelerated senescence, that is, apoptosis. $^{27}$

In the third type of stress, the injury is so harmful that cells cannot find a new steady state: the level of intracellular damage rises and global metabolic activity decreases so much that the cells cannot run their housekeeping pathways, and they die by necrosis. ${ }^{26}$

\section{HUMAN CELLS AND NONCYTOTOXIC STRESS}

In vivo, human cells are constantly exposed to various types and degrees of noncytotoxic stress of various nature and intensity. These types of stress include, for instance, UV irradiation of skin keratinocytes, fibroblasts, and melanocytes, effects of oxidized low density lipoproteins (LDL) on endothelial cells and smooth muscle cells, effects of urban pollution or tobacco smoke on lung pneumocytes and endothelial cells, effects of food oxidants on enterocytes, effects of repeated exposures of hepatocytes to ethanol, reactive oxygen species in skeletal myocytes during intense exercise or in inflammation processes as well as pathobiological hypoxia/reoxygenation processes. 
Two hypotheses have been put forward for the role of senescent cells in tissues: either senescent cells could accumulate in vivo in tissues, as shown for fibroblasts, ${ }^{28,29}$ using two different biomarkers, or the senescent cells are removed by immune cells or undergo apoptosis, thereby participating in the aging of tissues through the installation of a microinflammatory state or/and activation of neighboring cells. ${ }^{30}$

The accumulation of such senescent cells might be very slow in vivo under normal physiologic conditions. Nevertheless, exposure of cells to repeated or chronic nonlethal stress may participate in an increase in the accumulation of stress-induced senescent cells, thereby accelerating tissue aging. It is also suggested that local accumulation of such stress-induced senescent cells may be responsible for the alteration in tissue function, the differentiation status of neighboring cells, and may be a focus responsible for the start of pathological processes linked with aging. ${ }^{31}$

\section{IMMEDIATE AND LONG-TERM STRESS RESPONSES}

The immediate response to stimulations by growth factors, cytokines, and the immediate response to stresses, such as oxidative stress, ionizing radiation, osmotic stress, mechanical stress, hypoxia, heavy metals, and heat shock, have received much attention from many investigators. The long-term cellular response to stresses, from 48 hours to several weeks after stress or after stimulation by cytokines, has received much less attention. The development of proper experimental noncytotoxic models allowed the start of research programs on the long-term cellular response to noncytotoxic stresses or stimulations.

\section{LONG-TERM STRESS RESPONSES AND STRESS-INDUCED PREMATURE SENESCENCE}

Exposure of human skin fibroblasts to repeated noncytotoxic oxidative or UV stress triggers the nonreversible appearance of the biomarkers of cellular senescence, beginning 48-72 hours after the end of the stress.

First, morphologic studies showed that human diploid fibroblasts exposed to repeated sublethal stress under tert-butylhydroperoxide $(\mathrm{t}-\mathrm{BHP})^{32}$ or single $\mathrm{H}_{2} \mathrm{O}_{2}$ stress $^{33}$ display the phenotype of SIPS. The first studies were designed to follow cellular morphology after stress. This work was based on the description of the successive fibroblast morphotypes observed during in vitro aging (three successive mitotic fibroblasts followed by three successive postmitotic fibroblasts and a degenerative state of short existence). Various interpretations have been given about these seven successive morphotypes. Whatever this interpretation, many morphologists have been able to determine reproducibly the proportions of the various morphotypes in culture of fibroblasts at very low density, allowing spreading of cells. ${ }^{32,34-37}$ It is possible to discriminate human diploid fibroblasts into classes according to their morphology: a software able to sort fibroblast morphologies from CCD camera pictures could be developed (unpublished data). Using this type of classification, it was possible to show that after sublethal stress under UV light, t-BHP, ethanol, mitomycin C, 
A. Percentage of morphotypes (1) and S-A $\beta$-gal positive cells in each morphotype (2)

\begin{tabular}{|c|c|c|c|c|c|c|}
\hline \multirow{2}{*}{$\begin{array}{l}\text { Morpho- } \\
\text { types }\end{array}$} & \multicolumn{2}{|c|}{ Fibroblasts at early CPDs } & \multicolumn{2}{|c|}{ Fibroblasts at late CPDs } & \multicolumn{2}{|c|}{$\begin{array}{c}\text { Fibroblasts at early CPDs } \\
+5 \text { stresses under } 30 \mu \mathrm{M} \\
\text { t-BHP }\end{array}$} \\
\hline & (1) & (2) & (1) & (2) & (1) & (2) \\
\hline $\mathrm{I}$ & $2.0 \pm 0.2$ & $0.0 \pm 0.0$ & $2.3 \pm 0.7$ & $0.0 \pm 0.0$ & $0.6 \pm 1.0$ & $0.0 \pm 0.0$ \\
\hline II & $86.2 \pm 1.3$ & $2.8 \pm 0.3$ & $41.3 \pm 4.1$ & $20.6 \pm 7.8$ & $19.9 \pm 3.2$ & $35.3 \pm 13.8$ \\
\hline III & $10.1 \pm 1.2$ & $41.3 \pm 8.2$ & $46.5 \pm 5.6$ & $61.1 \pm 15.8$ & $54.5 \pm 6.1$ & $54.3 \pm 10.1$ \\
\hline IV & $1.3 \pm 0.5$ & $88.7 \pm 19.6$ & $7.9 \pm 2.1$ & $75.3 \pm 6.3$ & $21.2 \pm 3.7$ & $79.0 \pm 12.1$ \\
\hline $\mathrm{V}$ & $0.5 \pm 0.2$ & $83.3 \pm 18.9$ & $1.8 \pm 1.7$ & $94.4 \pm 9.6$ & $2.0 \pm 1.5$ & $93.3 \pm 11.6$ \\
\hline
\end{tabular}

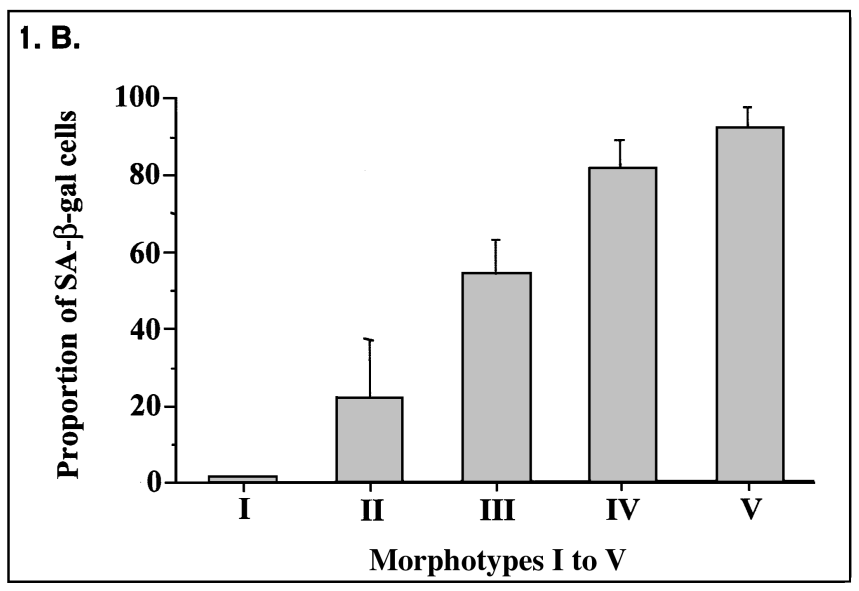

FIGURE 1. (A) Relationship between the fibroblast morphotypes and the senescenceassociated (S-A) $\beta$-gal activity in WI-38 fibroblasts at early CPDs (50\% of proliferative life span), at late CPDs ( $>95 \%$ of proliferative life span), or at early CPDs after exposure to 5 stresses under $30 \mu \mathrm{M}$ t-BHP performed as described earlier $(1 \mathrm{hr} /$ day for 5 days followed by a 48-hr recovery). ${ }^{39}$ Morphotype proportions: At 24 hours after seeding cells at a density of 700 cells per $\mathrm{cm}^{2}$, the proportions of the different morphotypes were recorded by two different examiners as previously described ${ }^{32}$ on 400 cells/dish. Results are mean values \pm SD of 4 different countings. Results are expressed as percentages of the total number of cells counted. Senescence-associated $\beta$-galactosidase activity (S-A $\beta$-gal): At 24 hours after seeding cells at a density of 700 cells per $\mathrm{cm}^{2}$, S-A $\beta$-gal was detected as described in reference 29. The proportions of cells positive for S-A $\beta$-gal are given as percentages of the number of cells of each morphotype and as mean values \pm SD in 4 different experiments (400 cells/experiment). S-A $\beta$-gal detection was never performed on confluent cells to avoid effects of confluency. (B) Average proportions of S-A $\beta$-gal positive cells for morphotype I to $\mathrm{V}$ in WI-38 fibroblasts. The average proportion of S-A $\beta$-gal positive cells is presented as the mean value obtained for each morphotype in fibroblasts at early CPDs, at late CPDs, and at early CPDs after 5 stresses under $30 \mu \mathrm{M}$ t-BHP. Morphotypes VI and VII were not presented because they represented less than $0.025 \%$ of the total population in these experimental conditions. 
and the like, the treated fibroblasts acquired the morphologic features of fibroblasts at late cumulative population doublings (CPDs). ${ }^{32,38}$ (For a review see ref. 31.) More recently, it was shown that the proportion of fibroblasts positive for senescence-associated $\beta$-galactosidase activity was increased after successive sublethal stresses under t-BHP ${ }^{39}$ after at least 2 days of recovery after the stresses.

\section{CORRELATION BETWEEN FIBROBLAST MORPHOTYPES AND SENESCENCE-ASSOCIATED $\beta$-GAL ACTIVITY IN SIPS}

The proportions of fibroblasts positive for senescence-associated $\beta$-galactosidase activity (S-A $\beta$-gal) were determined for each morphotype in cultures at early or late CPDs and after five successive exposures to $30 \mu \mathrm{M}$ t-BHP at early CPDs (FIG. 1) performed as described earlier ( 1 stress/day for 5 days +2 days of recovery). ${ }^{32,39}$ In all three situations, there were very few S-A $\beta$-gal positive cells in morphotypes I and II. Most morphotypes IV and V were positive for the S-A $\beta$-gal. Morphotypes III could be seen as intermediate morphotypes with a mean value of $52 \%$ positive cells among the three situations.

In all three situations, each morphotype displayed similar proportions of cells positive for S-A $\beta$-gal, whatever the relative proportion of the morphotype in the cultures. The only significant variation concerned morphotype II at late CPDs where a few more cells were positive for S-A $\beta$-gal than in young cultures.

\section{OTHER BIOMARKERS OF REPLICATIVE SENESCENCE IN SIPS}

After sublethal stresses under $30 \mu \mathrm{M}$ t-BHP, the cells developed other biomarkers of replicative senescence such as loss of replicative potential, elevation of cyclin-dependent kinase inhibitors $\mathrm{p} 21^{\text {Waf-1/SDI-1/Cip1 }}$, and failure to hyperphosphorylate the retinoblastoma protein. ${ }^{39,40}$ The common 4,977-bp mitochondrial DNA deletion was detected in WI-38 HDFs at late CPDs and at early CPDs after $30 \mu \mathrm{M}$ t-BHP stresses. ${ }^{39}$

The amounts of mRNAs transcribed from at least 15 genes specifically over- or underexpressed in normal senescence changed similarly in SIPS. ${ }^{39,41}$ Human fibroblasts stressed under $40 \%$ hyperoxia displayed accelerated senescence as judged by accelerated shortening of their telomeric sequences, growth arrest, lipofuscin accumulation, and mitochondrial respiration. Telomere shortening can trigger a senescence-like growth arrest via liberation of G-rich single-stranded DNA and activation of a DNA damage-response pathway dependent on p53 and its downstream effector p $21^{\text {waf- }-142}$. Retrovirally mediated stable transfection of mutants showed that the retinoblastoma protein is involved in the control of the appearance of the senescent phenotype and is responsible for the relocalization of focal adhesion proteins 72 hours after noncytotoxic stress under $\mathrm{H}_{2} \mathrm{O}_{2}$. De novo protein synthesis is necessary for the appearance of the senescent phenotype. ${ }^{43}$

Human normal melanocytes exposed to repeated sublethal doses of UV also express biomarkers of cellular senescence, namely, modifications in the regulation of the cell cycle as, for example, the prolonged stable overexpression of p21waf-1 44 as seen in fibroblasts undergoing SIPS. 
Taken together, these data strongly suggest that exposure of human proliferative cell types to repeated noncytotoxic stresses leads to SIPS provided the stress is intense enough in terms of concentration, duration, dose, or chronicity.

\section{SENESCENCE-ASSOCIATED $\beta$-GAL ACTIVITY AND MORPHOTYPE TRANSITION AFTER STIMULATION WITH IL-1 $\alpha$ AND TNF- $\alpha$}

Stimulations of fibroblasts with TNF- $\alpha$ or IL-1 $\alpha$ transiently increase the production of reactive oxygen species in human fibroblasts. It was shown that repeated stimulations of WI-38 fibroblasts with TNF- $\alpha$ or IL- $1 \alpha$ accelerate the transition from early to late fibroblast morphotypes. Such stimulations also increase the proportion of fibroblasts positive for the S-A $\beta$-gal. The involvement of reactive oxygen species was suggested by experiments in which the stimulations of fibroblasts with TNF- $\alpha$ or IL- $1 \alpha$ were performed in the presence of $N$-acetylcysteine, which increases the intracellular antioxidant potential. ${ }^{37,45} \mathrm{~N}$-acetylcysteine protected against stimulation-induced changes in the proportions of morphotypes and in the proportions of cells positive for $S$-A $\beta$-gal.

\section{ROLE OF ENERGETICS IN THE RESISTANCE TO STRESS-INDUCED PREMATURE SENESCENCE}

The thermodynamic theoretical model of aging described herein predicts that sublethal stress may induce SIPS. It also predicts that the level of global metabolic activity may protect the cells against SIPS and against cell death caused by lethal stress. Review and data in favor of this prediction as far as cell death is concerned have been presented. ${ }^{31,46}$ Preliminary results suggesting that substrates of the energy metabolism protect against SIPS were already published in references 34 and 35. In this paper, we provide more experimental arguments in favor on this hypothesis: two biomarkers of replicative senescence, that is, S-A $\beta$-gal and morphotype proportions, were analyzed after five successive sublethal stresses under $25 \mu \mathrm{M}$ t-BHP or $4 \% \mathrm{v} / \mathrm{v}$ ethanol diluted in PBS buffer ( 1 hour of stress/day for 5 days +48 hours of recovery) in the presence of D-glucose or vitamin E. D-glucose $(5 \mathrm{mM})$ was added in PBS buffer for each stress (1 hour), whereas vitamin E (1 mM) was present throughout the experiment beginning 24 hours before the first stress.

At 48 hours after t-BHP treatment, it was found that D-glucose and vitamin E protected the cells against the stress-induced decrease in the proportions of morphotype II and increase in morphotypes III to VI (FIG. 2A). After ethanol stresses, D-glucose similarly protected the cells against changes in the morphotype proportions, whereas vitamin E did not (FIG. 2B).

Similar experiments were carried out in which the proportions of cells positive for the S-A $\beta$-gal were determined at day 2 after the stresses. D-glucose had a protective effect against the increase in the proportion of positive cells observed after stress under ethanol and t-BHP. Vitamin E protected only against the stress under tBHP (FIG. 3).

From these experiments, it is suggested that D-glucose protects the cells against SIPS induced by several types of stress, whereas specific defense mechanisms, in 


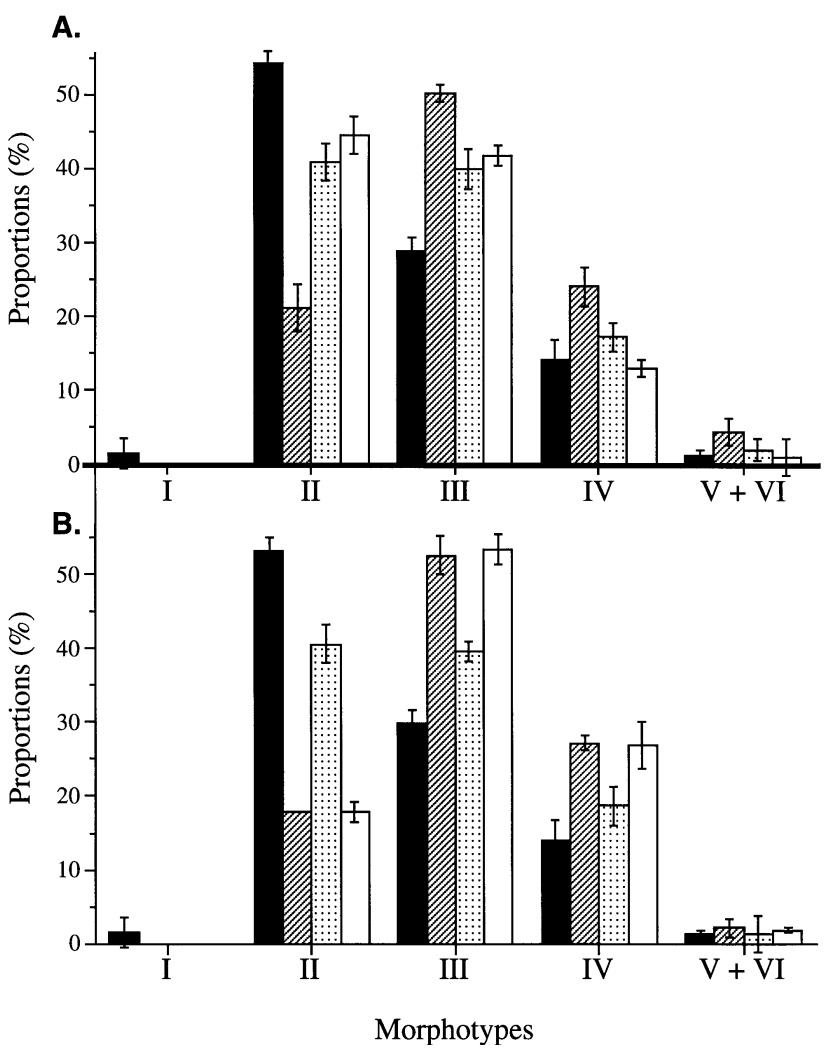

FIGURE 2. Effect of D-glucose and vitamin $E$ on the proportions of morphotypes obtained after 5 successive stresses under $25 \mu \mathrm{M}$ t-BHP (A) or $4 \%(\mathrm{v} / \mathrm{v})$ ethanol (B). Cells were exposed to the stressor for 1 hour for 5 consecutive days and were thereafter given 2 days of recovery after the stresses, as described earlier. ${ }^{39}$ Cells were plated at a density of 700 cells/ $\mathrm{cm}^{2}$ for 24 hours before morphotype determination performed as described earlier. ${ }^{32}$

this case antioxidant protection, protect only against specific stress, in this case oxidative stress. In fibroblasts, ethanol has been described as exerting fluidification effects on biological membranes. Ethanol also generates osmotic stress. ${ }^{47}$ These experimental arguments are in favor of the theoretical model, which predicts that SIPS is favored when the cellular energetic potential is decreased.

\section{CONCLUSION}

Corollaries of the second law of thermodynamics allow the proposal that all living systems have common energetic traits. In all of them, stress and/or aging results in lower energy flow and lower specific entropy production (weight-normalized entropy production), meaning lower global metabolic activity. 


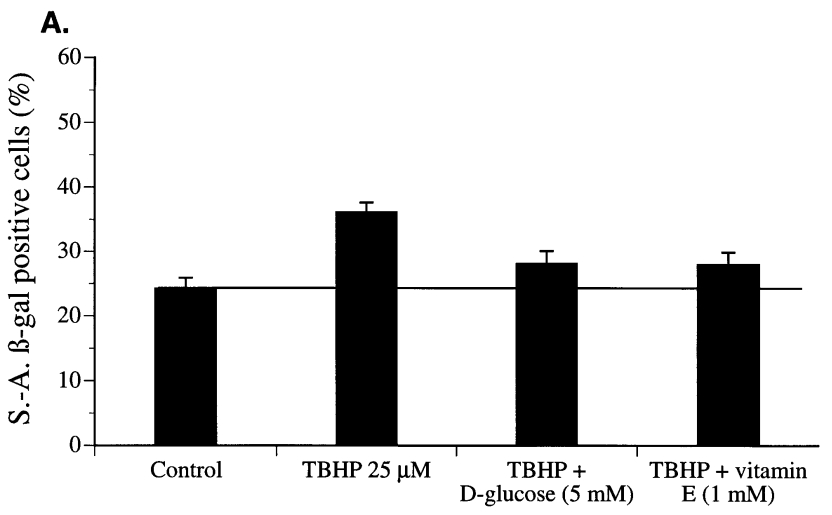

B.

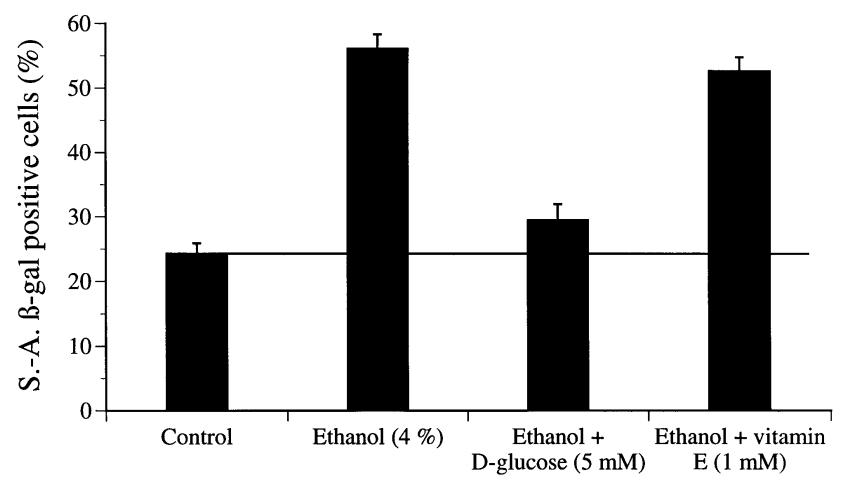

FIGURE 3. Effect of D-glucose and vitamin $E$ on the proportions of cells positive for the S-A $\beta$-gal obtained after 5 successive stresses under $25 \mu \mathrm{M}$ t-BHP (A) or $4 \%(\mathrm{v} / \mathrm{v})$ ethanol (B). Cells were exposed to the stressor for 1 hour for 5 consecutive days and were thereafter given 2 days of recovery after the stresses, as described earlier. ${ }^{39}$ Cells were plated at a density of 700 cells $/ \mathrm{cm}^{2}$ for 24 hours before histochemical detection of S-A $\beta$-gal performed as described earlier. ${ }^{29}$

Evolution resulted in cells that optimize their production and use of free energy in given ecologic conditions. Instabilities, however, can occur that can destabilize the biologic systems. ${ }^{21,22,26}$ As far as aging is concerned, cells can shift through a succession of steady states characterized by a higher level of damage and lower global metabolic activity, until a critical threshold is reached where the cells can no longer survive.

\section{ACKNOWLEDGMENTS}

C. Frippiat, J.-F. Dierick, F. Chainiaux, and P. Dumont have a fellowship of the FRIA, Belgium, and O. Toussaint from the FNRS, Belgium. The European Union 
Biomed and Health Research Programme, Concerted Action Programme 'Molgeron' (BMH1 CT94), and shared-cost action 'Genage' (BMH2 CT98), the Fulbright program, and the Belgian FRFC and FRSM allowed us to carry out this work.

\section{REFERENCES}

1. Selye, H. 1952. The Story of Adaptation Syndrome. Acta Inc. Medical Publishers. Montreal.

2. Selye, H. 1936. A syndrome produced by diverse noxious agents. Nature 138: 32 .

3. Selye, H. 1946. The general adaptation syndrome and the diseases of adaptation. J. Clin. Endocrinol. 6: 117-230.

4. BERCZI, I. 1998. The stress concept and neuroimmunoregulation in modern biology. Ann. N.Y. Acad. Sci. 851: 3-12.

5. Selye, H. 1976. Stress in Health and Disease. Butterworth. Boston.

6. CZAbo, S. 1998. Hans Selye and the development of the stress concept. Ann. N.Y. Acad. Sci. 851: 19-27.

7. LevitT, J. 1980. Responses of Plants to Environmental Stress. Vol 1. Academic Press. New York.

8. LARCHER, W. 1987. Streß bei Pflantzen. Naturwissenschaften 74: 158-167.

9. LichtenhaleR, H.K. 1998. The stress concept in plants: an introduction. Ann. N.Y. Acad. Sci. 851: 187-198.

10. Martin, G.M. 1997. Genetics and the pathobiology of ageing. Philos. Trans. R. Soc. Lond. B. Biol. Sci. 352: 1773-1780.

11. Kapahi, P., M.E. Boulton \& T.B. Kirkwood. 1999. Positive correlation between mammalian life span and cellular resistance to stress. Free Rad. Biol. Med. 26: 495500.

12. KIRKwood, T.B. 1997. The origins of human ageing. Philos. Trans. R. Soc. Lond. B. Biol. Sci. 352: 1765-1772.

13. Westendorp, R.G. \& T.B. KIRKWOOD. 1998. Human longevity at the cost of reproductive success. Nature 396: 743-746.

14. Kirkwood, T.B. \& A. Kowald. 1997. Network theory of aging. Exp. Gerontol. 32: 395-399.

15. ORgel, L.E. 1973. Ageing of clones of mammalian cells. Nature 243: 441-445.

16. Holliday, R. 1988. Towards a biological understanding of the ageing process. Perspect. Biol. Med. 32: 109-123.

17. Michiels, C., M. Raes, E. Pigeolet et al. 1990. Importance of a threshold for error accumulation in cell degenerative processes. Modulation of the threshold in a model of free radical-induced cell degeneration. Mech. Ageing Dev. 51: 41-54.

18. Remacle, J., D. Lambert, E. Pigeolet et al. 1992. Importance of the various antioxidant enzymes for the stability of cells. Confrontation between theoretical and experimental data. Biochem. J. 286: 41-46.

19. Denbeigh, K.G. 1951. The Thermodynamics of the Steady State. Methuen Ltd. London.

20. Prigogine, I. 1955. Thermodynamics of Irreversible Processes. John Wiley. New York.

21. SCHNEIDER, E.D. \& J.J. KAY. 1994. Life as a manifestation of the second law of thermodynamics. Mathl. Comput. Modelling 19: 25-48.

22. SCHNEIDER, E.D. \& J.J. KAY. 1995. Order from disorder: the thermodynamics of complexity in biology. In What Is Life: The Next Fifty Years. M. P. Murphy \& L.A. O'Neil, Eds.: 85-99. Cambridge University Press. Cambridge.

23. Glansdorff, P. \& I. Prigogine. 1971. Thermodynamics of Structure, Stability, and Fluctuations. Wiley Interscience. New York.

24. SCHNEIDER, E.D. 1988. Thermodynamics, ecological succession and natural selection: a common thread. In Entropy, Information, and Evolution: New Perspectives on Physical and Biological Evolution. B.H. Weber, D.J. Deprew \& J.D. Smith, Eds.: 107-138. MIT Press. Boston.

25. Toussaint, O. \& E.D. Schneider. 1998. The thermodynamics and evolution of complexity in biological systems. J. Comp. Physiol. Biochem. Part A 120A: 3-9. 
26. Toussaint, O., M. Raes \& J. Remacle. 1991. Aging as a multi-step process characterized by a lowering of entropy production leading the cell to a sequence of defined stage. Mech. Ageing Dev. 64: 45-64.

27. Remacle, J., M. Raes, O. Toussaint \& G. RaO. 1995. Low levels of reactive oxygen species as modulators of cell function. Mutation Res. 316: 103-122.

28. Bayreuther, K., P.I. Francz \& H.-G. Meinrath. 1991. The molecular biology of the terminal differentiation of dermel fibroblasts in the fibroblast stem cell system in vivo and in vitro. In Cell Culture Methods in Dermatological Research. Springer-Verlag. Heidelberg.

29. Dimri, G.P., X. LeE, G. Basile et al. 1995. A biomarker that identifies senescent cells in culture and in aging skin in vivo. Proc. Natl. Acad. Sci. USA 92: 9363-9367.

30. Giacomoni, P. \& P. D’Alessio. 1996. Skin ageing: the relevance of antioxidants. In Molecular Gerontology. S. Rattan \& O. Toussaint, Eds.: 177-192. Plenum Press. New York.

31. Toussaint, O. \& J. Remacle. 1996. Role of the cellular energetic metabolism in agerelated processes. In Molecular Gerontology. S. Rattan \& O. Toussaint, Eds.: 87109. Plenum Press. New York.

32. Toussaint, O., A. Houbion \& J. Remacle. 1992. Aging as a multi-step process characterized by a lowering of entropy production leading the cell to a sequence of defined stage II. Experimental results with cultivated cells. Mech. Ageing Dev. 65: 65-83.

33. Chen, Q. \& B. N. Ames. 1994. Senescence-like growth arrest induced by hydrogen peroxide in human diploid fibroblast F65 cells. Proc. Natl. Acad. Sci. USA 91: 4130-4134.

34. Toussaint, O., C. Michiels, M. Raes \& J. Remacle. 1995. Importance of the energetic factors in cellular ageing. Exp. Gerontol. 30: 1-22.

35. Toussaint, O., F. Eliaers, A. Houbion et al. 1995. Protective effect of EGb 761 and bilobalide on mortality and accelerated cellular ageing in stressful conditions. In Advances in Ginkgo Biloba Extract Research 4. Effect of Ginkgo Biloba Extracts (EGb 761) on Aging and Age-related Disorders. Y. Christen, Y. Courtois \& M.-T. Droy-Lefaix, Eds.: 1-16. Elsevier Publications. Paris.

36. Hamels, S., O. Toussaint, L. Le et al. 1995. AGO4432 fibroblasts also shift through seven successive morphotypes during in vitro ageing: comparison with WI-38 human foetal lung fibroblasts and HH-8 human skin fibroblasts. Arch. Int. Physiol. Biochem. Biophys. 103: B16.

37. Rodemann, H.P., K. Bayreuther, F. Dittmann et al. 1989. Selective enrichment and biochemical characterization of seven human skin fibroblasts cell types in vitro. Exp. Cell Res. 180: 84-93.

38. Toussaint, O., M. Raes, C. Michiels \& J. Remacle. 1998. Cellular response to stress: relationship with ageing and pathology. Medecine/Science. 14: 622-635.

39. Dumont, P., Q.M. Chen, M. Burton et al. 2000. Induction of replicative senescence biomarkers by sublethal oxidative stresses in normal human fibroblasts. Free Rad. Biol. Med. In press.

40. Chen, Q.M., J.C. BARtholomew, J. CAMPisi et al. 1998. Molecular analysis of $\mathrm{H}_{2} \mathrm{O}_{2}-$ induced senescent-like growth arrest in normal human fibroblasts: p53 and Rb control G1 arrest but not cell replication. Biochem. J. 332: 43-50.

41. Saretzki, G., J. Feng, T. von Zglinicki \& B. Villeponteau. 1998. Similar gene expression pattern in senescent and hyperoxic treated fibroblasts. J. Gerontol. 53A: B438-B442.

42. Von Zglinicki, T., G. Sarentzki, W. Döcke \& C. Lotze. 1995. Mild hyperoxia shortens telomeres and inhibits proliferation of fibroblasts, a model for senescence. Exp. Cell Res. 220: 186-213.

43. Chen, Q.M., S. Tu, V. CAtania et al. Induction of senescent morphology by $\mathrm{H}_{2} \mathrm{O}_{2}$ involves de novo synthesis, functional $\mathrm{Rb}$ Protein and redistribution of focal adhesion proteins. Submitted for publication.

44. Medrano, E.E., S. Im, F. Yang \& Z.A. Abdel-MaleK. 1995. Ultraviolet B induces G1 arrest in human melanocytes by prolonged inhibition of retinoblastoma protein phosphorylation associated with long-term expression of the p21Waf-1/SDI-1 protein. Cancer Res. 55: 4047-4052. 
45. Dumont, P., L. Balbeur, J. Remacle \& O. Toussaint. 2000. Appearance of biomarkers of in vitro ageing after successive stimulations of WI-38 fibroblasts with IL- $1 \alpha$ and TNF- $\alpha$ : senescence associated $\beta$-galactosidase activity and morphotype transition. J. Anat. In press.

46. Toussaint, O., A. Houbion \& J. Remacle. 1994. Effects of modulations of the energetical metabolism on the mortality and ageing of cultured cells. Biochim. Biophys. Acta 1186: 209-220.

47. BARnes, Y., S. Houser \& F.A. BARILE. 1990. Temporal effects of ethanol on growth, thymidine uptake, protein and collagen production in human foetal lung fibroblasts. Toxicol. in Vitro 4: 1-7. 